\title{
A IMPORTÂNCIA DO EXERCÍCIO FÍSICO NO PROCESSO DE ENVELHECIMENTO:
}

\section{uma revisão integrativa}

DOI: $10.48140 /$ digitaleditora.2020.002.10

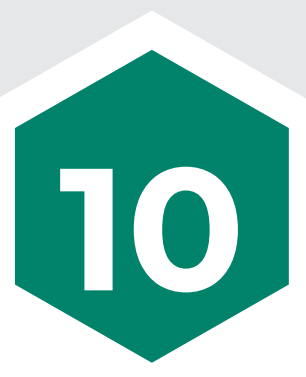

\section{RESUMO}

Objetivos: Avaliar os efeitos do exercício físico na saúde do idoso Métodos: Este trabalho tratou-se de uma revisão bibliográfica do tipo integrativa, onde a seleção dos estudos foi relizada na base de dados on-line: PubMed, entre os anos de 2010 a 2020 em português. Após o processo de filtragem dos estudos, obteve-se um total de 10 artigos.

Resultados: Estudos mostraram que o exercício físico é capaz de aumentar os escores no domínio cognitivo do idoso, melhora a flexibilidade, a capacidade funcional como por exemplo a marcha, além de reduzir a pressão arterial, glicemia e os fatores de risco cardiovascular, em consequência disto, uma melhor qualidade de vida para o idoso.

Conclusão: Foi possivel constatar que o exercício físico trouxe diversos efeitos positivos para os idosos, envolvendo a melhora da capacidade funcional para realizar as tarefas do dia-a-dia, diminuição de dores, como também, benefícios psicológicos e sociais.
Cássia Kaiane Braz Pinheiro

Graduanda em Educação Física pela AESPI - Ensino Superior do Piauí - Teresina - Piauí

iD https://orcid.org/0000 0002-0785-5576

Zenaide Castelo Branco Pereira da Silva

Graduando em Educação Física pela AESPI - Ensino Superior do Piauí - Teresina - Piauí

iD

https://orcid.org/00000002-8244-1058

\section{Tâmyack Alves de Macêdo}

Profissional de Educação Física, Especialista e Professor da Faculdade AESPI - Ensino Superior do Piauí - Teresina - Piauí

in https://orcid.org/00000002-8583-8325 


\section{THE IMPORTANCE OF PHYSICAL EXERCISE IN THE AGING PROCESS: an integrative review}

DOI: 10.48140/digitaleditora.2020.002.10

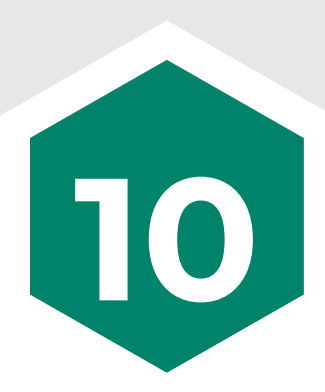

\section{ABSTRACT}

Recebido em: 10/12/2020

Aprovado em: 28/12/2020

Conflito de Interesse: não

Suporte Financeiro: não houve
Objectives: To evaluate the effects of physical exercise on the health of the elderly.

Methods: This work was a bibliographic review of the integrative type, where the selection of studies was relocated in the online database: PubMed, between the years 2010 to 2020 in Portuguese. After the filtering process of the studies, a total of 10 articles were obtained.

Results: Studies have shown that physical exercise is capable of increasing scores in the cognitive domain of the elderly, improving flexibility, functional capacity such as gait, in addition to reducing blood pressure, blood glucose and cardiovascular risk factors, as a result of this, a better quality of life for the elderly.

Conclusion: It was possible to verify that physical exercise brought several positive effects for the elderly, involving the improvement of functional capacity to perform day-to-day tasks, reduction of pain, as well as psychological and social benefits. 


\section{(8) \\ INTRODUÇÃO}

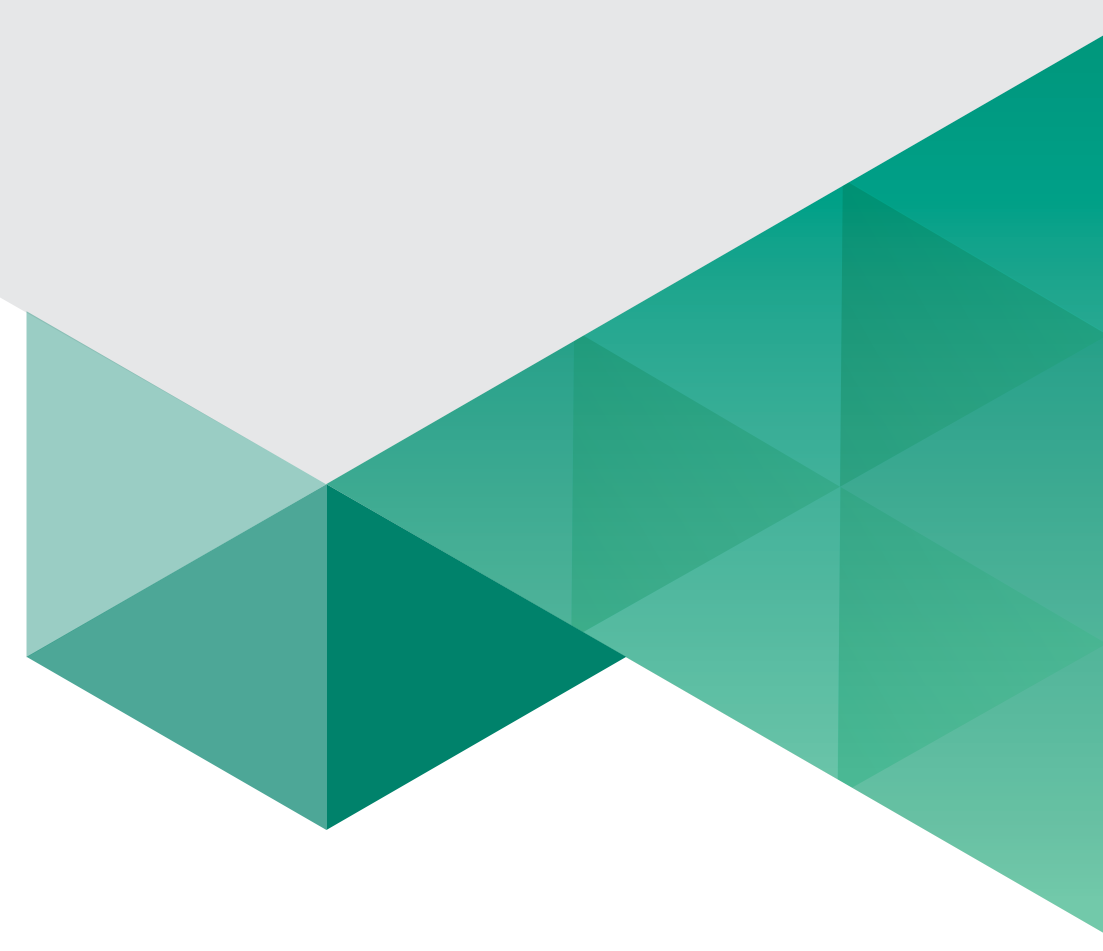

É considerado idoso todo indivíduo com idade igual e acima de 60 anos (OMS, 2005). A saúde da população idosa é um indicador de suas condições de vitalidade, ou seja, o delineamento dos indicadores de morbidades, mortalidade e qualidade de vida determina esse processo de saúde ou doença. O processo envelhecimento ocorre de maneira contínua é inevitável e irreversível, o mesmo causa ao organismo alterações e prejuízos em diversas funções. Essas alterações resultam em modificações fisiológicas, funcionais e psicológicas o que interfere diretamente na mobilidade, autonomia, qualidade de vida além de ser um fator que proporciona uma condição suscetível a depressão na população idosa. (MACIEL, 2010).

O processo de envelhecimento tem início a partir dos 30 anos de vida onde já começam as alterações do sistema nervoso central. O SNC é responsável por controlar as ações vitais e com isso as funções cognitivas, motricidades dentre outras são prejudicadas ao longo do tempo. Porém, há intervenções que tendem a desacelerar esse processo dentre essas intervenções estar a adesão do indivíduo a prática constante de exercício físico. O que é cada vez mais mencionada como ação de conter os avanços do processo de envelhecimento. Com base nisso tornou-se notadamente estabelecido que o exercício físico é eficaz tanto na prevenção quanto manutenção do declínio funcional do idoso. Os benéficos da prática incluem maior independência e autonomia, redução dos riscos de queda, autoestima elevada, aumento na expectativa de vida e consequentemente menor incidência de mortes. (SCIANNIS et al., 2019)

Segundo Bouchard et al., (2013) a maioria dos idosos não seguem as recomendações das agências de saúde para a prática de atividade física. Esse fato de inatividade física para a população idosa torna-se mais agravante pelo declínio fisiológico resultante do processo de envelhecimento. O que se sabe é que ainda não há comprovação por que os idosos não atendem a essas recomendações. O que pode deduzir é a não esclarecedora condição de que o exercício físico traga benefícios para a saúde e que de fato quais benefícios são esses.

As agências de saúde estabelecem níveis de atividade física para os idosos semelhante a população em geral onde recomendam o acúmulo 150 minutos de atividade física por semana de intensidade moderada. Além dos benefícios físicos é notório a melhora nos aspectos cognitivos que o exercício 
físico promove. A evolução na atenção auditiva e na velocidade do desenvolvimento intelectual pode estar relacionado a realização correta dos movimentos quando há solicitação para a execução do exercício proposto. Dessa forma manter os idosos ativos se faz importante tendo em vista, que as mortes e doenças crônicas não transmissíveis estão associado a inatividade física, afetando tanto a saúde como os gastos financeiros associados a ela. (TAYLOR, 2014).

Em um estudo conduzido por Villareal et al., (2011), mostrou fundamentos de que um programa de treinamento físico com múltiplos componentes (força, resistência muscular e flexibilidade) resulta em síntese de proteínas, redução no percentual de gorduras e melhora nas funções físicas. Além de melhorar a resistência, força e flexibilidade de idosos obesos melhoras também as habilidades simples como equilíbrio em ficar de pé, caminhar, sentar e levantar otimizando as atividades da vida diária e a aptidão física em geral.

Diante do exposto essa pesquisa tem por justificativa reunir elementos relevantes de maneira a levar informação e motivar esse público a adotar prática de atividade física como importante meio de ter um envelhecimento saudável. Portanto, esse trabalho tem como objetivo geral realizar uma revisão integrativa que avalia os efeitos positivos do exercício físico na saúde do idoso. Assim como objetivo específico avaliar através de evidências científicas os efeitos positivos do exercício físico sobre a funcionalidade do idoso, buscar referências das alterações morfofisiologia no processo de envelhecimento e comprovar os benefícios da adesão do exercício físico na saúde e bem-estar do idoso.

\section{METODOLOGIA | DELINEAMENTO DO ESTUDO}

O estudo caracteriza como uma pesquisa integrativa da literatura. De acordo com Souza et al., (2010) a revisão integrativa manifesta-se como um método em que possibilita a construção de um entendimento e a inclusão da aplicação de resultados de conteúdos significantes na prática.

\section{CRITÉRIOS DE INCLUSÃO}

Para elaboração do presente estudo, foram considerados: a) estudos que continham o público-alvo composto por indivíduos, de ambos os sexos, com idade igual ou superior a 60 anos; b) período de publicação entre 2010 a 2020; c) publicação com idioma em português; d) estudos experimentais em humanos que realizaram um método de mediação por meio do exercício físico; e) estudo que investigam, o efeito que o exercício físico provoca no idoso.

\section{CRITÉRIOS DE EXCLUSÃO}

Foram excluídos artigos com publicações fora do período de busca pré-estabelecida, estudos com indivíduos de idade inferior a 60 anos, pesquisas que fugissem do objetivo do estudo e publicações feitas com experimentos em ratos. 


\section{ESTRATÉGIA DE BUSCA}

A estratégia de busca nas bases de dados engloba estudos publicados nos últimos 10 anos (2010 - 2020), em apenas um idioma: Português.

Para elaboração do presente estudo, foram empregues uma das principais bases de dados da biblioteca virtual em Saúde (BVS): PubMed (National Library of Medicine).

Foram utilizados os seguintes termos de acordo com os descritores de ciências e saúde (DesCs) na língua inglesa, que continha como título: elderly people AND sedentary AND physical activity. Aplicando a forma boleana "AND" para a combinação dos termos citados.

\section{SELEÇÃO DOS ESTUDOS}

A seleção dos estudos foram realizadas, então, em cinco etapas. Sendo estas: 1a etapa - busca das publicações de acordo com os termos/descritores entre o ano de 1963-2020; 2a etapa - foram selecionados artigos com o ano de publicação entre (2010-2020); 3a etapa - processo de filtragem de textos completos e textos completos grátis; 4a etapa - seleção do idioma em português; 50a etapa: leitura dos títulos e resumos para exclusão dos artigos que não se encaixaram com o objetivo do estudo; 60 a etapa: leitura na íntegra dos 10 artigos selecionados na $5 \underline{a}$ etapa. Ficando assim, os que eram capazes de responder a todos os critérios de inclusão.

As buscas nas Bases de Dados ocorreram entre o período de 31 de outubro á 02 de novembro de 2020.

FIGURA 1. Processo de Filtragem e seleção dos artigos para a estruturação da pesquisa.

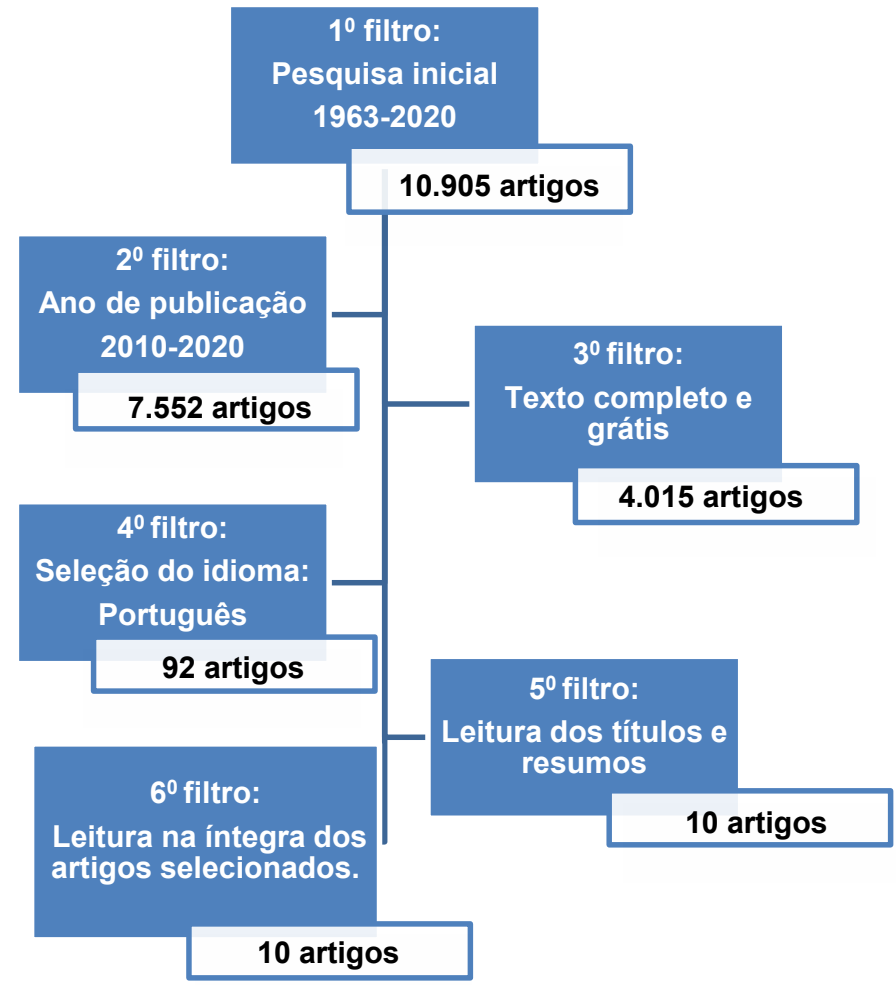




\section{RESULTADOS}

Nesta revisão integrativa da literatura foram incluídos 10 artigos. Na Base de dados da PubMed foram encontrados 10.905 artigos, destes foram excluídos 10.885 por não atenderem aos critérios de inclusão da pesquisa. Sendo estes: idioma (Português), tempo de publicação (artigos publicados nos últimos 10 anos, 2010 a 2020), tipo de estudo (artigos), base de dados (PubMed), artigos completos que investiguem os efeitos que o exercício físico provoca no idoso.

Na figura 01, através da combinação elderly people AND sedentary AND physical activity foram achados 10.905 recomendações, destes, 7.552 achavam-se no período de 2010 a 2020, da qual 4.015 se encontrava com o texto completo e grátis, sendo 92 de língua portuguesa e dentre esses, apenas 10 foram publicados na íntegra respondendo ao tempo de estudo e ao tema proposto.

FIGURA 1. Representação gráfica da associação dos descritores $(n=10)$.

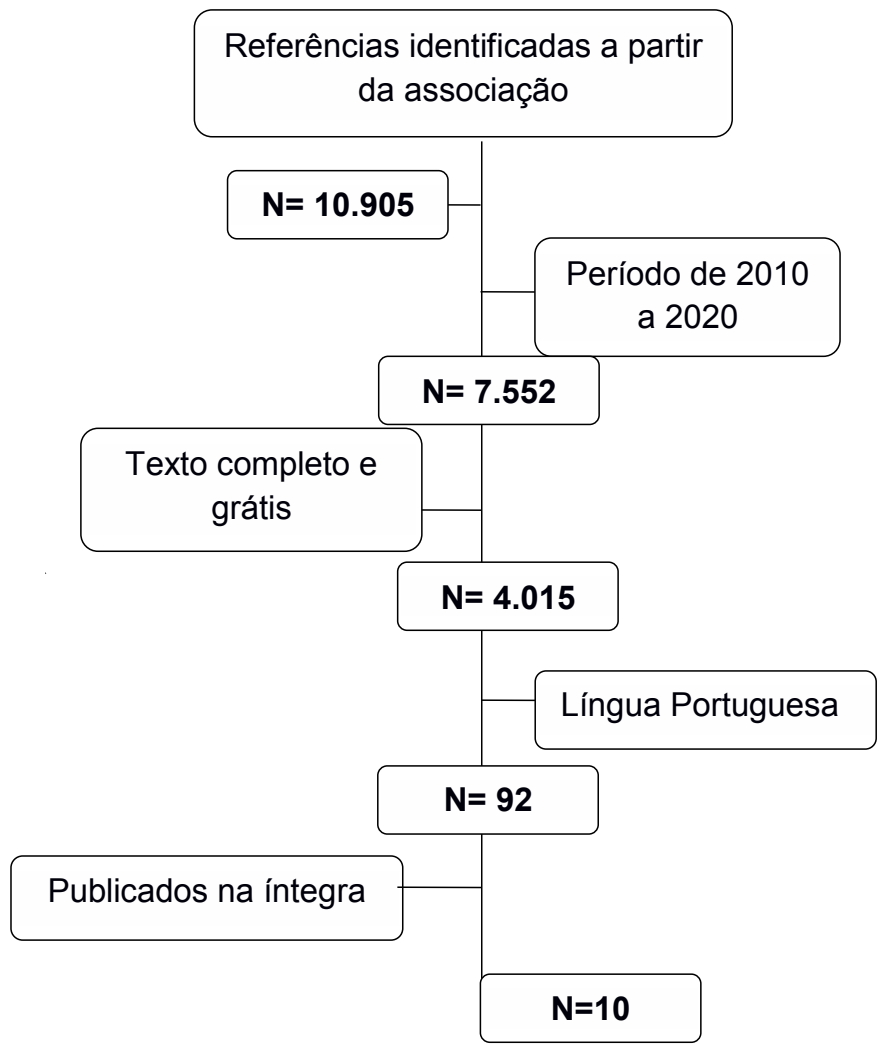

Fonte: Pesquisa realizada em base de dados. 
Na tabela 01, demonstra que entre os anos de publicação dos artigos, os anos de 2010, 2013 e 2020 tiveram a maior prevalência com 20\% cada. Nota-se que os anos 2012, 2014, 2018 e 2019 apresentam frações similares de estudos publicados no ano (10\%). Relacionado ao resultado demonstrado, os anos de 2011, 2015, 2016 e 2017 não tiveram nenhum número de artigo publicado. Observa-se na tabela que a pesquisa conseguiu obter um período de tempo apropriado para o tema, proporcionando uma comparação entre os anos.

Tabela 01: Organização dos artigos incluídos na amostra, referente ao ano de publicação.

\begin{tabular}{|c|c|c|}
\hline ANO DE PUBLCAÇÃO & N' ABSOLUTO & \% \\
\hline 2012 & 1 & $10 \%$ \\
\hline 2013 & 2 & $20 \%$ \\
\hline 2014 & 1 & $10 \%$ \\
\hline 2018 & 1 & $10 \%$ \\
\hline 2019 & 1 & $10 \%$ \\
\hline 2020 & 2 & $20 \%$ \\
\hline TOTAL & 10 & $100 \%$ \\
\hline
\end{tabular}

Fonte: Pesquisa realizada em base de dados.

Em relação aos periódicos, na tabela 02 pode-se perceber que a Ciência e Saúde coletiva obteve o maior número de artigos publicados, apresentando 50\% do total. Os demais periódicos, tiveram apenas um artigo utilizado na pesquisa, formando um total de 05 , representando (10\% cada) onde somando todos tiveram a sua grande contribuição para o desenvolvimento da pesquisa.

TABELA 02- Distribuição dos estudos incluídos na amostra, referente ao nome do periódico.

\begin{tabular}{|l|c|c|}
\hline \multicolumn{1}{|c|}{ NOME PERIÓDICO } & No ABSOLUTO & $\%$ \\
\hline Ciência e Saúde coletiva & 5 & $50 \%$ \\
\hline Revista saúde pública & 1 & $10 \%$ \\
\hline Einstein (São Paulo) & 1 & $10 \%$ \\
\hline Arquivos Brasileiro de Cardiologia & 1 & $10 \%$ \\
\hline Cadernos de Saúde Pública & 1 & $10 \%$ \\
\hline Revista Brasileira Epidemiologia & 1 & $10 \%$ \\
\hline TOTAL & 10 & $100 \%$ \\
\hline
\end{tabular}

A tabela 03 refere-se a organização da pesquisa de acordo com os autores e anos de publicação, esta distribuição acontece da seguinte maneira: autor/ano; tipo de estudo; objetivo de cada estudo e os resultados que foram encontrados sendo necessário para a construção do estudo. 
TABELA 03 - Refere-se a organização da pesquisa de acordo com os autores e anos de publicação, esta distribuição acontece da seguinte maneira: autor/ano; tipo de estudo; objetivo de cada estudo e os resultados que foram encontrados sendo necessário para a construção do estudo.

\begin{tabular}{|c|c|c|c|}
\hline $\begin{array}{l}\text { AUTOR/ } \\
\text { ANO }\end{array}$ & TIPO DE ESTUDO & OBJETIVO & RESULTADOS \\
\hline $\begin{array}{l}\text { Cassiano., } \\
\text { et al } 2020\end{array}$ & $\begin{array}{l}\text { Trata-se de um estudo } \\
\text { longitudinal (incidência), } \\
\text { prospectivo, do tipo quase } \\
\text { experimental. }\end{array}$ & $\begin{array}{l}\text { Avaliar o efeito do exercício físi- } \\
\text { co sobre o risco cardiovascular, } \\
\text { qualidade de vida e presença } \\
\text { de sintomas depressivos em } \\
\text { idosos hipertensos. }\end{array}$ & $\begin{array}{l}\text { Apresentou-se redução no valores médios } \\
\text { de colesterol total e LDL, redução de } 4,2 \% \\
\text { de sintomas depressivos, além da redução } \\
\text { dos fatores de risco cardiovascular. Tendo } \\
\text { assim, uma melhor qualidade de vida. }\end{array}$ \\
\hline $\begin{array}{l}\text { Bobbet., } \\
\text { et al } 2018\end{array}$ & $\begin{array}{l}\text { Trata-se de um estudo } \\
\text { transversal (prevalência), } \\
\text { com abordagem compa- } \\
\text { rativa. }\end{array}$ & $\begin{array}{l}\text { Avaliar a presença de dor crôni- } \\
\text { ca em idosos atendidos pela } \\
\text { Unidade de atenção primária, } \\
\text { comparando os praticantes da } \\
\text { ginástica chinesa e idosos se- } \\
\text { dentários. }\end{array}$ & $\begin{array}{l}\text { Os resultados obtiveram a presença de } \\
\text { dor maior nos idosos ativos, porém com } \\
\text { menos impedimento para a realização de } \\
\text { atividades quando comparados ao grupo } \\
\text { de sedentários. notou-se também que } \\
\text { idosos ativos tem percepção mais positiva } \\
\text { da saúde. }\end{array}$ \\
\hline $\begin{array}{l}\text { Mourão., } \\
\text { et al } 2013\end{array}$ & $\begin{array}{l}\text { Trata-se de um estudo de } \\
\text { prevalência ou transversal. }\end{array}$ & $\begin{array}{l}\text { Analisar o nível de atividade } \\
\text { física dos idosos no transporte } \\
\text { e lazer e fatores associados. }\end{array}$ & $\begin{array}{l}\text { A insuficiência ativa foi em maioria nos } \\
\text { idosos com idade avançada, sendo as mu- } \\
\text { lheres as mais insatisfeitas. Os relatos de } \\
\text { saúde estavam associado aos que pratica- } \\
\text { vam atividade física, sendo a caminhada a } \\
\text { mais preferida. }\end{array}$ \\
\hline $\begin{array}{l}\text { Oliveira., } \\
\text { et al } 2019\end{array}$ & $\begin{array}{l}\text { Trata-se de um estudo } \\
\text { epidemiológico descritivo, } \\
\text { de corte observacional e } \\
\text { transversal. }\end{array}$ & $\begin{array}{l}\text { Avaliar o nível de atividade } \\
\text { física e o estado cognitivo de } \\
\text { idosos. }\end{array}$ & $\begin{array}{l}\text { Verificou-se diferença significativa para os } \\
\text { idoso fisicamente ativos com maiores esco- } \\
\text { res no domínio cognitivo quando compara- } \\
\text { dos aos de menor nível de atividade física. }\end{array}$ \\
\hline $\begin{array}{l}\text { Roma., et } \\
\text { al } 2013\end{array}$ & $\begin{array}{l}\text { Ensaio clínico randomi- } \\
\text { zado. }\end{array}$ & $\begin{array}{l}\text { Compara os efeitos sobre a } \\
\text { aptidão física e funcionalidade } \\
\text { de idosos em dois programa de } \\
\text { atividade física supervisiona- } \\
\text { das: aeróbio e resistido. }\end{array}$ & $\begin{array}{l}\text { Os resultados do estudo mostrou melhora } \\
\text { da flexibilidade no GR e que o GA teve } \\
\text { melhora da capacidade funcional. Ambos } \\
\text { os grupos contribuirão positivamente para } \\
\text { aptidão física dos idosos. }\end{array}$ \\
\hline $\begin{array}{l}\text { Zaitune., } \\
\text { et al } 2010\end{array}$ & $\begin{array}{l}\text { Trata-se de estudo trans- } \\
\text { versal/prevalência. }\end{array}$ & $\begin{array}{l}\text { Analisar a prevalência da prá- } \\
\text { tica de atividade física global e } \\
\text { de lazer em idosos e seus fato- } \\
\text { res associados. }\end{array}$ & $\begin{array}{l}\text { A prevalência de ativo foi em maioria nas } \\
\text { mulheres, em lazer teve baixa prevalência } \\
\text { em ambos os sexos. No geral a prevalência } \\
\text { de atividade global foi para idoso jovens } \\
\text { sendo atividade física ocupacional. }\end{array}$ \\
\hline $\begin{array}{l}\text { Ribeiro., } \\
\text { et al } 2012\end{array}$ & $\begin{array}{l}\text { Trata-se de um estudo } \\
\text { epidemiológico descritivo, } \\
\text { de corte observacional e } \\
\text { transversal. }\end{array}$ & $\begin{array}{l}\text { Investigar as relações entre os } \\
\text { níveis de forças dos membros } \\
\text { inferiores e superiores e a prá- } \\
\text { tica regular de exercício físico } \\
\text { e atividade da vida diária de } \\
\text { mulheres idosas recrutadas na } \\
\text { comunidade. }\end{array}$ & $\begin{array}{l}\text { Os resultados do estudo apontam que as } \\
\text { medianas de força de pressão manual e do } \\
\text { n0 de ADV preservadas estavam mais altas } \\
\text { nas ativas do que nas sedentárias. As ido- } \\
\text { sas com mais de } 80 \text { e anos e baixa renda } \\
\text { eram as mais sedentárias e que apresenta- } \\
\text { vam menor força de pressão. Estando asso- } \\
\text { ciado a perda na atividade de vida diária. }\end{array}$ \\
\hline $\begin{array}{l}\text { Costa., } \\
\text { et al } \\
2020\end{array}$ & $\begin{array}{l}\text { Trata-se de estudo trans- } \\
\text { versal/prevalência. }\end{array}$ & $\begin{array}{l}\text { Avaliar a associação da síndro- } \\
\text { me metabólica com a atividade } \\
\text { física e as condições socioecô- } \\
\text { nomicas de idosos não institu- } \\
\text { cionalizados. }\end{array}$ & $\begin{array}{l}\text { Os resultados apontam a prevalência de } \\
\text { síndrome metabólica (SM) maior nos ido- } \\
\text { sos que não realizavam atividade física. } \\
\text { Idosos ativos possuem 33\% menos chances } \\
\text { de desenvolver a (SM) quando compara- } \\
\text { dos aos que não estavam ativos. Mostrou } \\
\text { também que indivíduos escolarizados tem } \\
\text { menos chances de adquirir a SM do que os } \\
\text { não escolarizados. }\end{array}$ \\
\hline
\end{tabular}




\begin{tabular}{|c|c|c|c|}
\hline $\begin{array}{l}\text { Queiroz., } \\
\text { et al } 2014\end{array}$ & $\begin{array}{l}\text { Estudo de prevalência(- } \\
\text { transversal) }\end{array}$ & $\begin{array}{l}\text { Descrever a inatividade física } \\
\text { de idosos e suas associações } \\
\text { com fatores sociodemográfico, } \\
\text { estilo de vida e condições de } \\
\text { saúde, em idosos do nordeste } \\
\text { brasileiro. }\end{array}$ & $\begin{array}{l}\text { Os resultados da pesquisa mostra preva- } \\
\text { lência significativa de inatividade física em } \\
\text { idosos acima de } 80 \text { anos ausência de ocu- } \\
\text { pação e dependência funcional. }\end{array}$ \\
\hline $\begin{array}{l}\text { Monteiro., } \\
\text { et al } 2010\end{array}$ & $\begin{array}{l}\text { Ensaio Clínico randomi- } \\
\text { zado. }\end{array}$ & $\begin{array}{l}\text { Analisar o efeito da intervenção } \\
\text { de } 13 \text { semanas de exercícios } \\
\text { aeróbios sobre a pressão arte- } \\
\text { rial , índice de massa corpórea } \\
\text { e a glicemia em idosas com } \\
\text { diabetes tipo } 2 \text {. }\end{array}$ & $\begin{array}{l}\text { Os resultados apontam que } 13 \text { semanas de } \\
\text { treino aeróbio foi capaz de reduzir signifi- } \\
\text { cativamente os valores de pressão arterial } \\
\text { e glicemia de idosas diabéticas. Não foram } \\
\text { encontradas reduções significativas no IMC } \\
\text { após o treinamento aeróbico em ambos os } \\
\text { grupos. }\end{array}$ \\
\hline
\end{tabular}

Fonte: pesquisa realizada em base de dados

\section{DISCUSSÃO}

As informações analisadas abordam questões relevantes sobre nível de atividade física e saúde dos idosos. Como foi visto no estudo conduzido por Zaitune,. et al (2010) que identificou a prevalência de idosos ativos e muito ativo se deu em maioria nas mulheres o que esta associado ao fato das mulheres realizarem mais atividades doméstica e de deslocamento comparado aos homens. Já no contexto geral os idosos mais jovens entre 60 a 79 anos são mais ativos nas atividades ocupacional enquanto que em atividades de lazer houve baixa prevalência em ambos os sexos. Entretanto, os idosos de maior grau de escolaridade, brancos e melhor renda familiar mostrou maior frequência nas atividades física de lazer.

Em um estudo similar de Mourão,. et al (2013) ao analisar o nível de atividade física em idosos revelou predomínio de insuficientemente ativo os idosos de maior idade relatando estarem insatisfeito com a saúde, essa autopercepção de saúde indica o grau de quanto ativo é esse idoso. Os autores apontam resultados semelhantes em que a insuficiência ativa no lazer ocorre com maior prevalência em idosos de baixa poder econômico e menor grau de escolaridade.

Corroborando com os autores anteriores Queiroz,. et al (2014) associa a redução da atividade física ao processo de envelhecimento sendo predominante em idosos de idade mais avançada sem trabalho e não alfabetizados. Essas condições de inatividade se associa a idade, ausência de ocupação e dependência funcional, essa ausência ocupacional compromete a capacidade física do idoso.

No ensaio clínico randomizado realizado por Roma,. et al (2013) ao comparar o treino resistido e aeróbio sobre a capacidade física e funcionalidade do idosos sedentário, constatou melhoras nas qualidade física analisada e que a flexibilidade e teste de sentar e levantar tiveram melhoras significativas em resposta ao treino resistido. È válido ressaltar que a flexibilidade é uma qualidade física fundamental para funcionalidade e a perda nos níveis está associada ao risco de lesões osteomusculares. Os resultados também apontam melhora no equilíbrio e velocidade da marcha com treino aeróbio revelando que tanto o treino resistido quanto aeróbio influência positivamente na funcionalidade e capacidade física de indivíduos idosos.

Ribeiro., et al (2012) também evidenciam em seu estudo que níveis de força para pressão manual e número de atividade física diária preservada foi significativamente mais alta em idosas ativas comparada a idosas sedentárias. Ressaltando que os níveis de força manual baixos é um importante indicador de sacorpenia. A inatividade física, o menores níveis de força de pressão e diminuição na velocidade da marcha estão ligado a um menor desempenho funcional.

A redução da capacidade funcional oriundas da inatividade física precede o aparecimento de doenças cronicas não transmissíveis. Isso pôde ser visto no estudo de Cassiano,. et al (2020) onde a dislipidemia 
esteve presente em 58,3\% da amostra e o sedentarismo 45,8\%. Porém, foi possível perceber que após a intervenção de um programa de exercício físico a dislipidemia teve uma redução significativa, além de haver redução nos sintomas de depressão que se deu pelo fato da atividade de lazer ameniza o stress e melhorar a socialização entre os idosos.

Outra condição associada a inatividade física são as alterações cognitiva que acompanham o processo de envelhecimento. O estudo de Oliveira,. et al (2019) ao examinar o nível de atividade física sobre o estado cognitivo de idosos notou o escore de cognição elevado nos idosos mais ativos quando comparado aos sedentários. Os resultados mostraram que $60 \%$ da amostra relatou boa percepção de saúde e os que fizeram essa afirmação eram os idosos ativo e muito ativo. Os autores apontam que o declínio cognitivo esta relacionado ao aumento do tecido adiposo pois esse aumento esta ligado a processos inflamatórios em vários tecidos do organismo, dentre eles os da região cerebral. Esse processo inflamatório causa morte dos neurônios o que gera prejuízos nas funções cognitiva. Porém o exercício físico promove a neurogênese e angiogênese por meio do fluxo sanguíneo direcionada a região cerebral além da redução no percentual de gorduras o que reduz os processos inflamatórios dos tecidos cerebrais.

É evidenciado que prática regular de exercício físico promove melhoras na saúde da população idosa em vários pontos, isso pôde ser visto no estudo de Bobo,. et al (2018) onde avaliou a presença de dor cronica em idosos sedentário comparado a praticantes de ginástica chinesa (Lian Gong). Os resultados revelam que ambos os grupos apresentaram queixas de dores osteomusculares porém, os idosos ativos relataram não ter empecilho para realizar suas atividades de vida diária sendo os ativos os que menos faziam uso de medicações além de relatarem percepção mais positiva de saúde.

Dentre as comorbidades síndrome metabólica é a que mais acometem os idosos. No estudo de Costa, et al ( 2020) quando verificou que a síndrome metabólica esteve presente em $40,1 \%$ da amostra examinada a pressão arterial foi a mais prevalente. E o que foi notado é que a predominância das síndromes metabólica estava entre os idosos que não apresentavam níveis de atividade física e mais presente nas pessoas de menor renda e baixa escolaridade.

Monteiro,. et al (2010) mostra em seu estudo que um programa de exercício aeróbio com duração de 13 semanas foi capaz de reduzir significativamente os valores de pressão arterial e glicemia de idosas diabéticas. Comprovando a eficácia do exercício físico em promover redução aos fatores de risco associado a doenças cardiovasculares. 


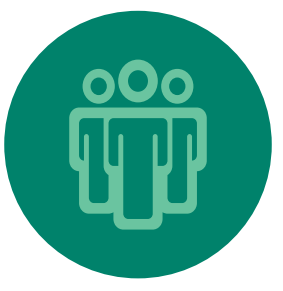

\section{CONCLUSÃO}

Nesse estudo verificou-se que durante o processo de envelhecimento, acontece no organismo diversas mudanças funcionais relacionadas a capacidades físicas, fisiológicas, sociais e cognitivas que são capazes de sofrerem o processo de retardamento por meio da prática de exercícios físicos.

Sendo assim, essas considerações efetuadas em relação aos efeitos positivos, nos levam a observar o fundamental papel que o exercício físico desempenha na vida destes idosos, contribuindo consideravelmente no processo de envelhecimento, para que os motivos nefastos deste processo sejam capazes de ser vencidos e, o idoso consiga atravessar essa etapa da melhor forma possivel, tendo hábitos mais saudáveis e uma boa qualidade de vida.

Pode-se concluir então que a prática de exercícios físicos traz inúmeros efeitos positivos, na qual indicam tanto benefícios físicos, como melhora considerável na capacidade funcional para realizar as tarefas do dia-a-dia, redução das dores, diminuição dos fatores de risco casdiovascular, glicemia e pressão arterial, bem como, benefícios sociais e psicológicos, tanto no convívio com outras pessoas como também no aumento da auto-estima, baixando assim, os índices de depressão. Confirmando assim, que o exercício físico traz efeitos positivos para os idosos em todos os sentidos, proporcionando aos mesmos, uma vida mais saudável e dinâmica que colabora para uma velhice mais sadia e, uma melhor qualidade de vida para os idosos. 


\section{REFERÊNCIAS}

BOUCHARD, Danielle R. et al. Pilot study: can older inactive adults learn how to reach the required intensity of physical activity guideline?. Clinical interventions in aging, v. 8, p. 501, 2013.

BOBBO, Vanessa Cristina Dias et al. Saúde, dor e atividades de vida diária entre idosos praticantes de Lian Gong e sedentários. Ciência \& Saúde Coletiva, v. 23, p. 1151-1158, 2018.

CASSIANO, Andressa do Nascimento et al. Efeitos do exercício físico sobre o risco cardiovascular e qualidade de vida em idosos hipertensos. Ciência \& Saúde Coletiva, v. 25, p. 2203-2212, 2020.

COSTA, Ana Cristina de Oliveira; DUARTE, Yeda Aparecida de Oliveira; ANDRADE, Fabíola Bof de. Síndrome metabólica: inatividade física e desigualdades socioeconômicas entre idosos brasileiros não institucionalizados. Revista Brasileira de Epidemiologia, v. 23, p. e200046, 2020.

MACIEL, Marcos Gonçalves. "Atividade física e funcionalidade do idoso." Motriz: Revista de Educação Física 16.4 (2010): 1024-1032.

MOURAO, Ana Raquel de Carvalho et al. Atividade física de idosos relacionada ao transporte e lazer, Maceió, Brasil. Revista de Saúde Pública, v. 47, p. 1112-1122, 2013

MONTEIRO, Luciana Zaranza et al. Redução da pressão arterial, da IMC e da glicose após treinamento aeróbico em idosas com diabete tipo 2. Arquivos Brasileiros de Cardiologia, v. 95, n. 5, p. 563-570, 2010.

OLIVEIRA, Daniel Vicentini de et al. O nível de atividade física como um fator interveniente no estado cognitivo de idosos da atenção básica à saúde. Ciência \& Saúde Coletiva, v. 24, p. 4163-4170, 2019.

QUEIROZ, Bruno Morbeck de et al. Inatividade física em idosos não institucionalizados: estudo de base populacional. Ciência \& Saúde Coletiva, v. 19, p. 3489-3496, 2014.

ROMA, Maria Fernanda Bottino et al. Efeitos das atividades físicas resistida e aeróbia em idosos em relação à aptidão física e à funcionalidade: ensaio clínico prospectivo. Einstein (São Paulo), v. 11, n. 2, p. 153-157, 2013.

RIBEIRO, Luciana Helena Martins; NERI, Anita Liberalesso. Exercícios físicos, força muscular e atividades de vida diária em mulheres idosas. Ciência \& Saúde Coletiva, v. 17, n. 8, p. 2169-2180, 2012.

SCIANNI, Aline Alvim, et al. "Efeitos do exercício físico no sistema nervoso do indivíduo idoso e suas consequências funcionais." Revista Brasileira de Ciências do Esporte 41.1 (2019): 81-95.

SOUZA, Marcela Tavares, et al. "Revisão integrativa: o que é e como fazer." Einsten (São Paulo) vol.8 no.1 Jan/Mar. 2010.

TAYLOR, Denise. "Physical activity is medicine for older adults." Postgraduate medical journal 90.1059 (2014): 26-32.

VILLAREAL, Dennis T., et al. "Regular multicomponent exercise increases physical fitness and muscle protein anabolism in frail, obese, older adults." Obesity 19.2 (2011): 312-318. 